Article

\title{
An FPGA Implementation of a Convolutional Auto-Encoder
}

\author{
Wei Zhao ${ }^{1}$, Zuchen Jia ${ }^{1}{ }^{(1)}$, Xiaosong Wei ${ }^{1}$ and Hai Wang ${ }^{2, *}$ \\ 1 Key Laboratory of Electronic Equipment Structure Design, Ministry of Education, Xidian University, \\ Xi'an 710071, China; weizhao@xidian.edu.cn (W.Z.); xdckjzc@139.com (Z.J.); winthor666@gmail.com (X.W.) \\ 2 School of Aerospace Science and Technology, Xidian University, Xi'an 710071, China \\ * Correspondence: wanghai@mail.xidian.edu.cn; Tel.: +86-029-8820-3115
}

Received: 24 February 2018; Accepted: 20 March 2018; Published: 27 March 2018

\begin{abstract}
In order to simplify the hardware design and reduce the resource requirements, this paper proposes a novel implementation of a convolutional auto-encoder (CAE) in a field programmable gate array (FPGA). Instead of the traditional framework realized in a layer-by-layer way, we designed a new periodic layer-multiplexing framework for CAE. Only one layer is introduced and periodically reused to establish the network, which consumes fewer hardware resources. Moreover, by fixing the number of channels, this framework can be applicable to an image of arbitrary size. Furthermore, to effectively improve the speed of convolution calculation, the parallel convolution method is used based on shift registers. Experimental results show that the proposed CAE framework achieves good performance in image compression. It can be observed that our CAE framework has advantages in resources occupation, operation speed, and power consumption, indicating great potential for application in digital signal processing.
\end{abstract}

Keywords: convolutional auto-encoder; neural network; image compression; FPGA

\section{Introduction}

Convolutional auto-encoder (CAE), a kind of convolutional neural network (CNN), adopts an unsupervised learning algorithm for encoding [1-5]. David E. Rumelhart first proposed the concept of an auto-encoder [6] and employed it to process data with large dimensions, which promoted the development of neural networks. In 2006, Hinton et al. [7] improved the original shallow auto-encoder and proposed the concept of a deep learning neural network as well as its training strategy, which can be used in the signal processing field for applications such as feature extraction [8], image compression [9-11], classification [12,13], image denoising [14], prediction [15], and so on. Wang et al. [16] proposed a rapid 3D feature learning method named a convolutional auto-encoder extreme learning machine (CAE-ELM), and the features extracted were superior to other previous deep learning methods. In [17], a three-layer multilayer perceptron structure was presented for image compression, which verified the applicability of the neural network in the field of image compression. Kim et al. [18] performed sonar image noise reduction with the CAE and obtained sonar images of superior quality with only a single continuous image. However, there is the same problem that the above applications running on CPU are all inefficient and time-consuming. To address this problem, GPU has been employed to accelerate the neural network [19,20], but the power consumption of GPU is too large.

A field programmable gate array (FPGA) breaks through the sequential execution mode of general-purpose processors, and is able to implement a neural network with high speed. In [21,22], two deep convolutional neural network (DCNN) accelerators were proposed which optimized the energy efficiency of the system by reducing the complexity of data transmission. Zhou et al. [23] used 
a fixed-point data type to represent parameters for computation based on FPGA, which achieved better performance and lower resource usage. To further enhance the performance, some researchers exploited the inherent parallelism of neural networks and utilized the multi-hardware and multi-product unit on FPGA to realize the network framework [24,25]. From the above references [21-25], we conclude that it is promising to accelerate neural networks by employing FPGA, which can make full use of the parallel processing characteristic of FPGA and the parallel computing framework of the neural network. However, the finite resource of FPGA makes it difficult to realize a neural network on a large scale.

To enhance the processing speed and reduce the power consumption and resource occupation, we propose a novel CAE using a periodic layer-multiplexing method, which periodically reuses only one layer to establish the network. On the one hand, by fixing the number of channels, this framework can be applicable to images of arbitrary size. On the other hand, to effectively improve the speed of convolution calculation, the parallel convolution method is used based on shift registers. The image compression is taken as an example to evaluate the performance of the proposed CAE framework. Experimental results show that our framework has advantages in terms of resource occupation, operational speed, and power consumption.

The rest of the paper is arranged as follows. In Section 2, the CAE framework and its key techniques are described in detail. In Section 3, experimental results and discussion are given. Finally, some conclusions are drawn in Section 4.

\section{FPGA Implementation of the CAE}

In essence, $\mathrm{CAE}$ is a multilayer $\mathrm{CNN}$ including convolution layers and pooling layers. The model of CAE used is shown in Figure 1, where the image compression is taken as an example. The convolution layers are mainly used to extract the features of inputs and the neurons in these layers share the weight parameters. The pooling layers are used to retain the main features and reduce the parameters, which also prevent overfitting and improve the generalization ability of the model. The upsampling process is performed to obtain the image in original resolution, which can be considered the inverse process of pooling.

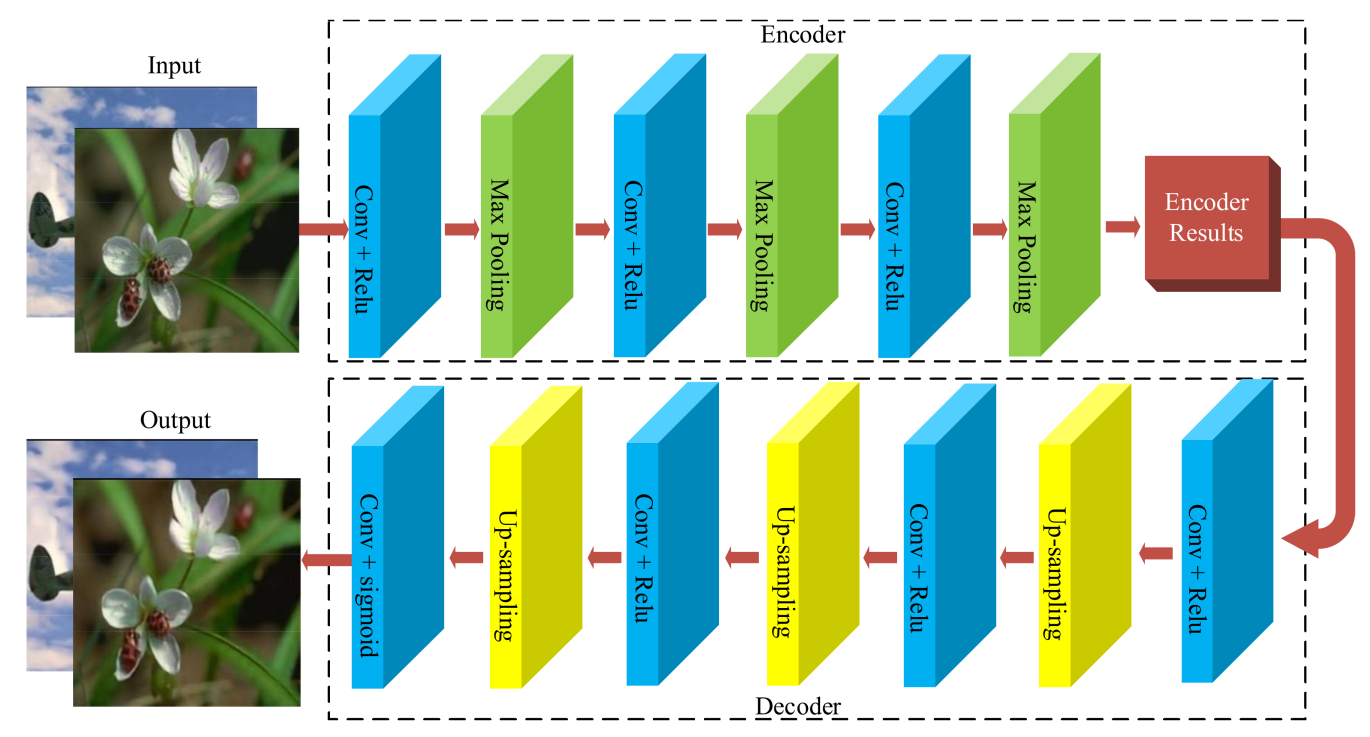

Figure 1. Classical model of a convolutional auto-encoder (CAE).

The model is divided into two parts: an encoder and a decoder. The encoder contains three convolutional layers and three pooling layers. The ReLU function and the maximum pooling operation are used. The decoder, which performs the reverse operation of the encoder, consists of four 
convolution layers and three upsampling layers. The ReLU function and the sigmoid function are used in the first three and the last convolution layers, respectively.

From Figure 1, it can be seen that the layers of CAE have a certain regularity and periodicity, so we implement their functions by proposing the periodic layer-multiplexing framework based on FPGA. The hardware framework and the relative key techniques are described as follows.

\subsection{Hardware Framework}

As shown in Figure 2, the encoder part of the proposed CAE framework contains five main modules: zero padding, a channel distributor, convolutional encoding, a channel arbitrator, and an output controller. The detailed explanation is as follows:

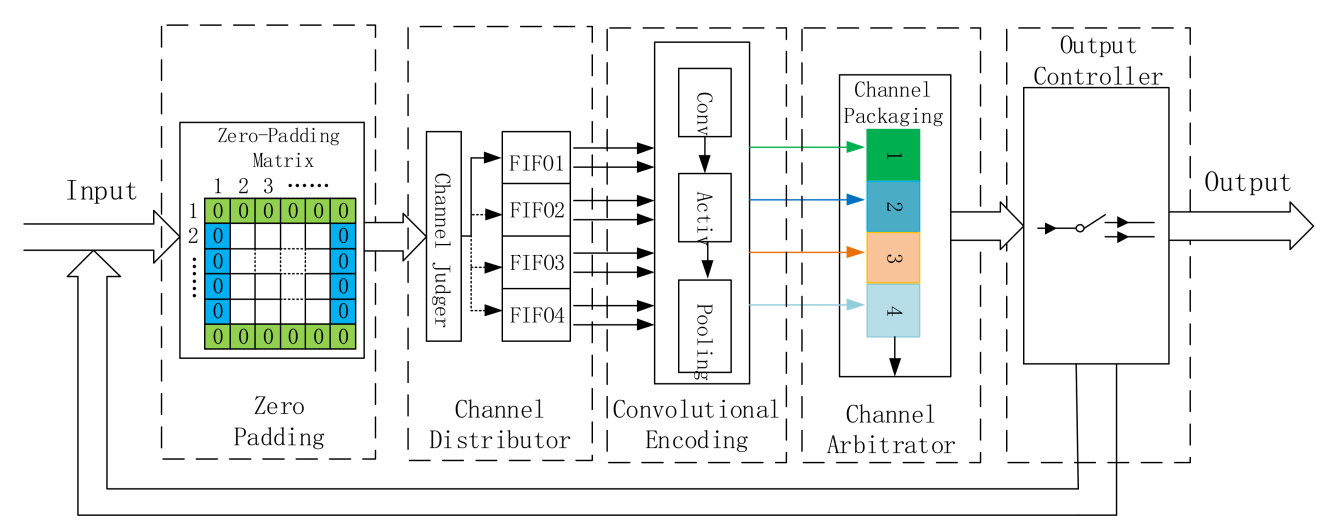

Figure 2. The encoder of the proposed CAE hardware framework. The different colors marked in the zero padding module indicate different filling methods: the first and the last row are filled all zeroes, while the intermediate matrix is padded zeroes only at the beginning and the end of each row. The different colors marked in the channel arbitrator module represent the corresponding four channels for packaging.

\section{(1) Zero Padding Module}

To prevent losing the boundary information during the convolution operation, the zero padding module is employed to fill zeros around the input matrix. The obtained matrix is defined as the zeropadding matrix in this paper.

(2) Channel Distributor Module

The zero-padding matrix is sent to the channel distributor module, which contains a channel judger and four first-in, first-out (FIFO) memories. The channel judger interleaves every two singlechannel data points and then sends them to the FIFOs in sequence. The FIFOs buffer the interleaved data, and then divide them into eight channels.

\section{(3) Convolutional Encoding Module}

The work process of this module is summarized as follows: First, the convolution layers in this module execute the convolution calculation in eight channels at the same time. The convolution kernels, obtained by training CAE, are stored directly in the internal LUT resources. By this means, the frequent data interaction between FPGA and external memory is avoided, and the transmission latency is reduced significantly. Then, in order to introduce nonlinear factors after the convolution calculation, activation functions are integrated into the convolution layers. Specifically, the ReLU function is employed for fast convergence and high accuracy. Finally, the maximum pooling is chosen to retain the main features. The first set of eight-channel data is transformed to the three-channel data after the pooling operation, while the subsequent sets of eight-channel data are transformed to the four-channel data. 
(4) Channel Arbiter Module

The output data of the convolutional encoding module are orderly packaged as the single-channel data, and then sent to the next module by the channel arbiter module for judgment.

(5) Output Controller Module

This module is used to judge whether the data stream is processed completely. The above modules process continuously until the ending data of the zero-padding matrix are handled.

\subsection{Design of Convolutional Encoding Module}

Convolutional encoding is the most important module in the framework; therefore, its structure is further discussed below. To effectively enhance the compatibility of our framework and control the utilization of hardware resources reasonably, the number of input channels is fixed to eight rather than the maximum number of neurons in a certain layer. To realize the simultaneous convolution calculation of eight channels, the parallel channels method is developed by caching the input data.

The operational process of the method is shown in Figure 3. Four identical processing elements (PEs) are used to process the input data, and the ones with identical sequence number are the same. These PEs are independent of each other so they can operate simultaneously. Therefore, all the convolutional results of the eight channels can be calculated simultaneously by inputting the data in sequence. The RAM1 and RAM2 are used to cache the last two-channel data of every eight inputs, which can avoid loading repetition of the input and improve the efficiency of data utilization. In Figure $3,8 \times i+j(i=1, \ldots,\lceil\mathrm{M} / 8\rceil-1$ and $j=1,2, \ldots, 8)$ represents the index of channel. Meanwhile, $M$ represents the total number of rows of zero-padding matrix, and the symbol \lceil\rceil represents a rounding-up operation.

At stage 1 (the black box in Figure 3), the first two inputs are transmitted to the first two channels of PE1, while the next two inputs are transmitted simultaneously to the other channels of PE1 and the first two channels of PE2. The next two inputs are provided to both the last two channels of PE2 and the first two channels of PE3. The last two inputs are sent to the last two channels of PE3 and RAM1 at the same time. The RAM1 is employed to buffer data, so that these data can be recycled without re-entering from outside. As shown in Figure 3, once the first set of eight-channel data from the zero-padding matrix has been processed at stage 1, the subsequent set of eight-channel data is sent to PEs at stage 2 (red box in Figure 3). At stage 2, the data in RAM1 and the subsequent inputs are sent to PEs, and a similar operation is undertaken as that at stage 1.

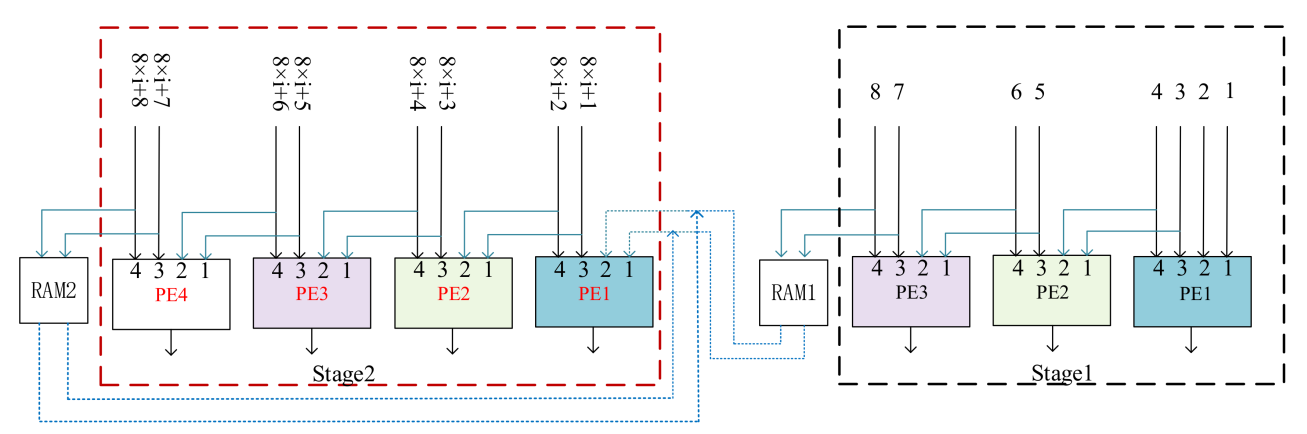

Figure 3. Parallel channels method by caching input data.

\subsection{Design of $P E$}

The PEs employed by the periodic layer-multiplexing framework are used to implement the operation of multiplication and addition required by convolution calculation. To reduce the time consumption of convolution calculation, a parallel convolution method based on shift registers is presented for the design of PE. 
There are four input channels in one PE, and the 16-bit unsigned numbers in the four channels can be inputted to the corresponding 32-bit shift registers per clock cycle. These 32-bit shift registers act as the convolution window, which can slide arbitrarily. We first assume that the data are inputted to these shift registers immediately after reset. Then, the convolution calculations begin in the second clock cycle. A $4 \times 4$ convolution window of the PE is shown as the colorful square in Figure 4 .

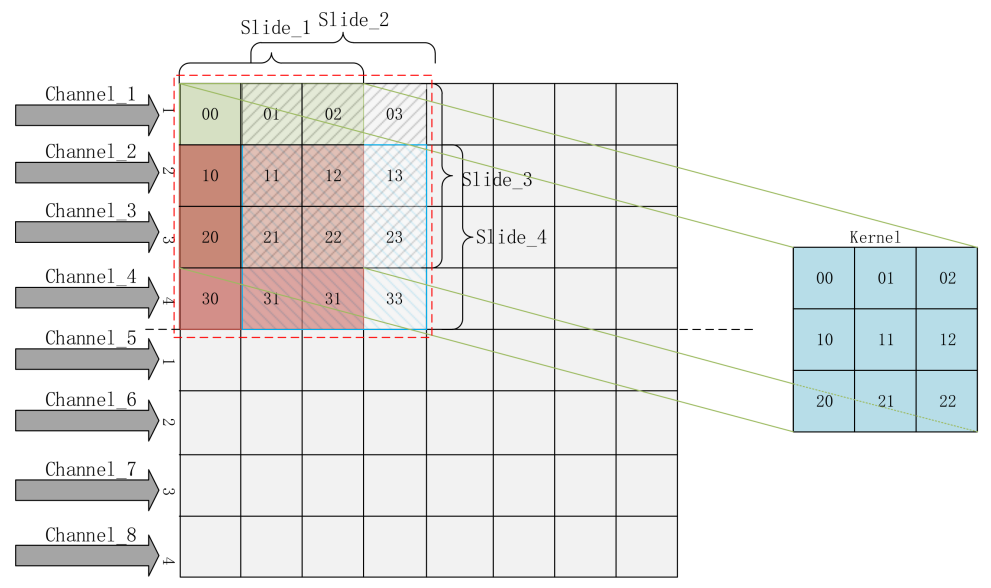

Figure 4. The $4 \times 4$ convolution window (the red dotted box) contains four $3 \times 3$ sub-windows: Slide_1, Slide_2, Slide_3, and Slide_4, which are marked with different colors or streaks, respectively.

For storage convenience, a total of nine 8-bit signed numbers of each convolution kernel are combined in a row to form an integrated data with a width of 72 bits. When the convolution calculation starts, the convolution window begin to slide so that convolution operations can be performed in the four sub-windows simultaneously under one clock cycle. Then every 8-bit convolution kernel is multiplied by the data in the shift register, as shown in Figure 5. After that, the outputs of multipliers are obtained under the third clock cycle. All these outputs of multipliers are summed and exported under the fourth clock cycle. Some nonlinear factors are introduced into these results by operating the ReLU activation function. The outputs of all the ReLU functions are transmitted to the pooling module, and then three comparators in this module start to perform comparison operation among the four values and output the maximum, which consumes two clock cycles.

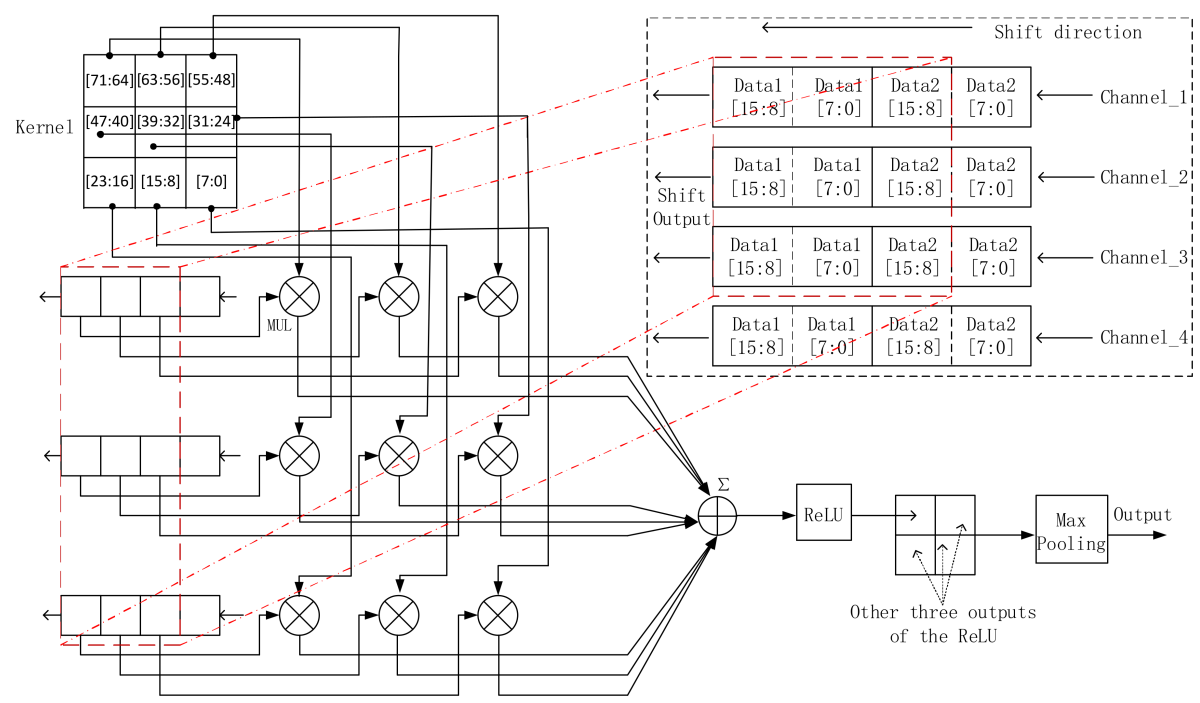

Figure 5. Convolution operation process in PE. 
The pseudo-code of the operation is as follows (algorithm 1):

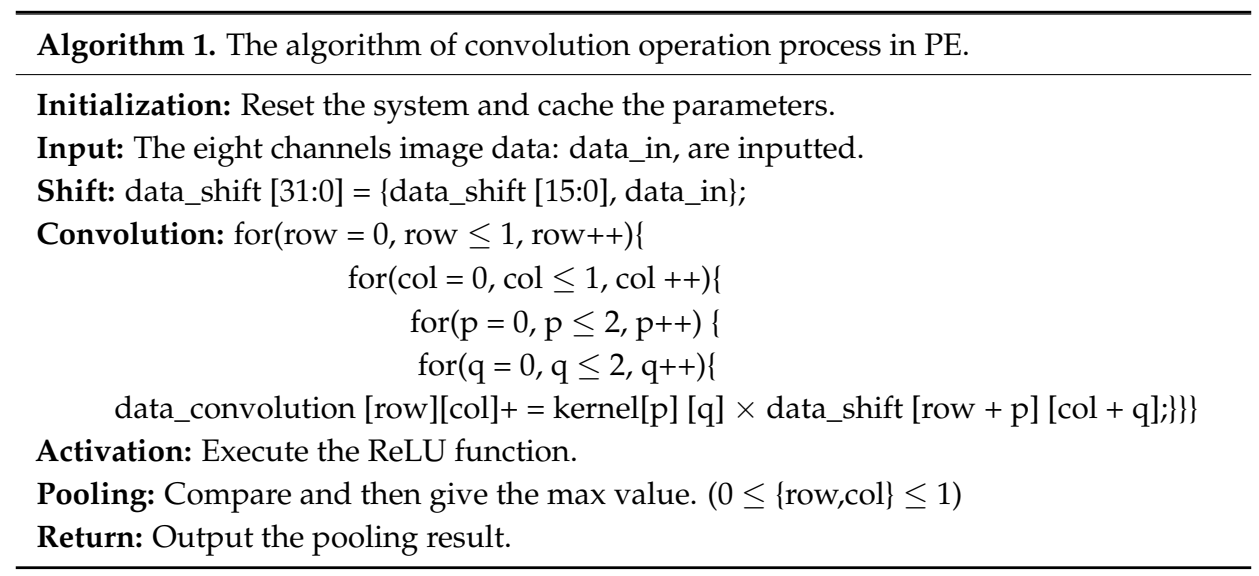

\section{Experimental Results and Discussion}

In order to test the performance of the proposed implementation of CAE, an application of image compression is carried out. We use Keras, which is a Python deep learning library, to establish the framework. There are 7000 digital images with a dimension of $256 \times 256 \times 3$ employed to train the network and obtain the parameters. During training, the epoch of iteration is chosen to be 1500, while the batch size is chosen to be 4 . The binary_crossentropy cost function, which can avoid the training process being too slow, is selected as the loss function. It has a non-negative character and when the real output is close to the expected output, the function value is close to 0 . The learning rate is chosen to be Adadelta, which is an adaptive learning rate method. The test starts once the training is finished. In this paper, 1000 additional images are employed to verify the performance of image compression based on the proposed CAE.

In this experiment, the Xilinx KCU105 evaluation board (Xilinx, San Jose, CA, USA) with a XCKU040-2FFVA1156E FPGA chip (Xilinx, San Jose, CA, USA) is employed to act as the hardware platform. The photograph of the implemented prototype is shown in Figure 6, from which we can see that the evaluation board is connected to a computer by JTAG cable and PCI-e bus. FPGA is programmed and debugged through the JTAG cable, while the data are transferred between the computer and FPGA through the PCI-e bus.

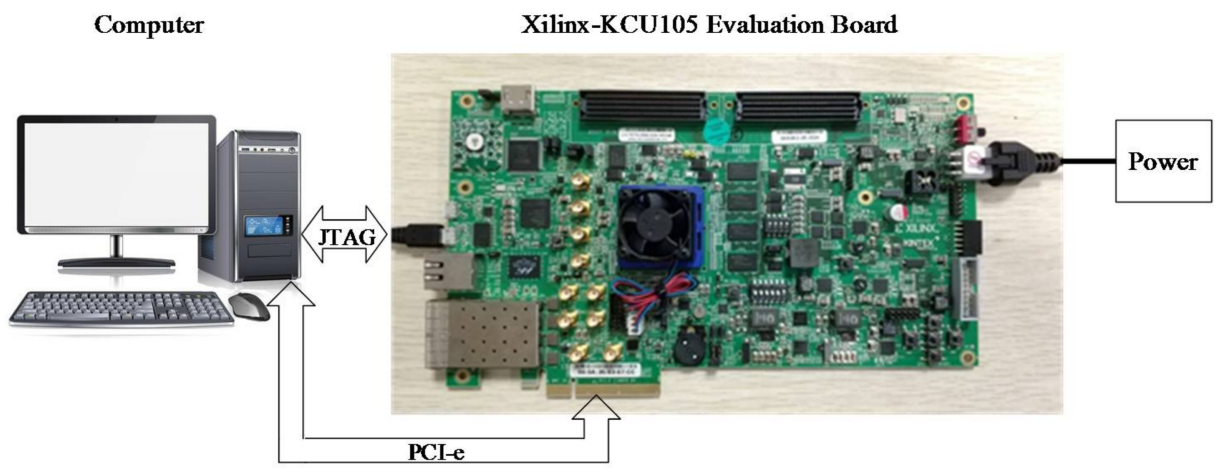

Figure 6. The hardware platform.

In order to measure the performance of our compression framework, we can use some typical metrics, such as compression ratio, Peak Signal to Noise Ratio (PSNR), and Multi-scale structural similarity (MS-SSIM). The image compression ratio obtained from a CAE can be expressed as

$$
\mathrm{S}=\mathrm{Ni} / \mathrm{No},
$$


where $\mathrm{Ni}$ is the neuron numbers of input layer and No represents the neuron numbers of the encoding output. According to Equation (1), the image compression ratio for our CAE is calculated. PSNR [26] is often used to assess the quality of signal reconstruction. The formula of PSNR is as follows:

$$
\operatorname{PSNR}=10 \cdot \log _{10}\left(\frac{\mathrm{MAX}}{\mathrm{MSE}}\right)
$$

where $\mathrm{MAX}_{\mathrm{I}}$ represents the maximum value of the image color. MSE is the mean square error between the original image and the reconstructed image. MSE is expressed as shown below:

$$
\text { MSE }=\frac{1}{m n} \sum_{i=0}^{m-1} \sum_{j=0}^{n-1}\|I(i, j)-K(i, j)\|^{2}
$$

where $m$ and $n$ represent the length and the width of an image. Meanwhile, I(i,j) and $K(i, j)$ represent the pixel values of the original and reconstructed images, respectively. MS-SSIM index is another image quality evaluation algorithm that is based on the assumption that the human visual system is highly adapted for extracting structural information from the scene, and therefore a measure of structural similarity can provide a good approximation of perceived image quality [27].

We randomly selected 12 images in the image dataset for testing, and the original and reconstructed images are shown in Figure 7. The numerical parameters of PSNR and MS-SSIM are also shown under the corresponding image in Figure 7. By comparison, we can conclude that our implementation of CAE based on FPGA works well and has good image compression and

\begin{tabular}{|c|c|c|c|c|c|c|}
\hline & Image 1 & Image 2 & Image 3 & Image 4 & Image 5 & Image 6 \\
\hline \multicolumn{7}{|l|}{$\begin{array}{c}\text { Original } \\
\text { Image }\end{array}$} \\
\hline \multicolumn{7}{|l|}{$\begin{array}{l}\text { Reconstructed } \\
\text { Image }\end{array}$} \\
\hline PSNR & 31.1273 & 38.5543 & 28.9924 & 37.2793 & 33.1653 & 35.3047 \\
\hline \multirow[t]{2}{*}{ MS-SIMM } & 0.9439 & 0.9737 & 0.8540 & 0.9541 & 0.9561 & 0.9670 \\
\hline & Image 7 & Image 8 & Image 9 & Image 10 & Image 11 & Image 12 \\
\hline \multicolumn{7}{|l|}{$\begin{array}{l}\text { Original } \\
\text { Image }\end{array}$} \\
\hline $\begin{array}{l}\text { constructe } \\
\text { Image }\end{array}$ & & & & & & \\
\hline PSNR & 30.9547 & 28.8112 & 34.4868 & 36.5804 & 29.2187 & 34.4206 \\
\hline MS-SIMM & 0.9414 & 0.9434 & 0.9562 & 0.9776 & 0.9294 & 0.9790 \\
\hline
\end{tabular}
reconstruction effects.

Figure 7. Original and reconstructed images. 
By using Verilog hardware description language, our hardware procedure is synthesized and implemented using the Xilinx Vivado2016.2 design suite (Xilinx, San Jose, CA, USA), which provides a full solution for Xilinx programmable devices. According to the device utilization summary given by the design suite, the utilization of the main hardware resources is shown in Table 1. With the numbers of network layers and weight parameters set at 13 and 68,227, respectively, we can see that the main resources, such as LUT and FF, take a very small percentage.

Table 1. Device utilization summary.

\begin{tabular}{cccc}
\hline Resource & Utilization & Available & Utilization $\%$ \\
\hline LUT & 22,441 & 242,400 & 9.26 \\
FF & 27,100 & 484,800 & 5.59 \\
BRAM & 55.50 & 600 & 9.25 \\
IO & 120 & 520 & 23.08 \\
BUFG & 8 & 480 & 1.67 \\
MMCM & 2 & 10 & 20 \\
\hline
\end{tabular}

Our CPU platform is Intel i7-6700K CPU (Intel, Santa Clara, CA, USA) at 4 GHz with a 16 GB RAM. The GPU platform is NVIDIA GTX TITAN Xp (NVIDIA, Santa Clara, CA, USA). A $150 \mathrm{MHz}$ system clock is used for the framework on FPGA. In the experiment, "time.clock()" instruction of Keras is used to obtain the computing time while running on either CPU or GPU. The computing time of FPGA is calculated by counting the number of clock cycles consumed from input to output. As for the power consumptions of GPU, CPU, and FPGA, they are acquired from the "nvidia-smi" command provided by NVIDIA, the electro-instruments, and the power analyzer integrated in Vivado2016.2, respectively. A performance comparison of FPGA, CPU, and GPU is shown in Table 2.

Table 2. Comparison of performance when running on the FPGA, CPU, and GPU.

\begin{tabular}{cccc}
\hline Performances & FPGA & CPU & GPU \\
\hline Computing time (ms) & 15.73 & 115.29 & 13.65 \\
Power consumption (W) & 2.762 & 32 & 44 \\
Computing performance (GOPS) & 73.18 & 3.25 & 76.38 \\
Computing performance per Watt (GOPS/W) & 26.49 & 0.101 & 1.73 \\
\hline
\end{tabular}

As the experimental results show, in terms of compression speed, it consumes 15.73 ms running on FPGA and $115.29 \mathrm{~ms}$ running on CPU. In terms of the computing performance per watt, it is 26.49 GOPS/W running on FPGA, which is far beyond that of CPU and GPU.

\section{Conclusions}

An implementation of CAE based on FPGA is presented in this paper, which newly introduces a periodic layer-multiplexing framework. The encoder part of the proposed CAE framework, which is similar to the decoder, contains five modules, zero padding, a channel distributor, convolutional encoding, a channel arbitrator, and an output controller. In order to enhance the compatibility of the framework and further enhance the speed of convolution calculation, a parallel channels method caching input data and a parallel convolution method based on shift registers are developed, respectively. To evaluate the performance of the CAE framework, an example of image compression is carried out. The experimental results of image compression show that the framework works well and takes up fewer hardware resources, especially as the resource utilization of LUT is just $9.26 \%$. In terms of the compression speed, it consumes 15.73 ms running on FPGA and 115.29 ms running on CPU. In terms of the computing performance per watt, it is 26.49 GOPS/W running on FPGA, which is far beyond that of CPU and GPU. In future work, more multiplexing techniques, like encoder multiplexing, can be adopted to enhance the CAE performance. 
Author Contributions: Hai Wang, Wei Zhao, and Zuchen Jia conceived and designed the experiments; Zuchen Jia and Xiaosong Wei performed the experiments; Wei Zhao and Zuchen Jia analyzed the data; Xiaosong Wei contributed analysis tools; Hai Wang, Wei Zhao, and Zuchen Jia wrote the paper.

Conflicts of Interest: The authors declare no conflict of interest.

\section{References}

1. Iqbal, M.S. Unsupervised Multi-modal Learning. In Advances in Artificial Intelligence; Lecture Notes in Artificial Intelligence; Springer: Berlin, Germany, 2015; Volume 9091, pp. 343-346.

2. Nugent, A.; Kenyon, G.; Porter, R. Unsupervised adaptation to improve fault tolerance of neural network classifiers. In Proceedings of the 2004 NASA/DoD Conference on Evolvable Hardware, Seattle, WA, USA, 26 June 2004; IEEE Computer Society: Los Alamitos, CA, USA, 2004; pp. 146-149.

3. Guest, E. Face image analysis by unsupervised learning. Trends Cogn. Sci. 2002, 6, 145. (In English) [CrossRef]

4. Guan, Y.; Ghorbani, A.A.; Belacel, N. An unsupervised clustering algorithm for intrusion detection. In Advances in Artificial Intelligence, Proceedings; Lecture Notes in Artificial Intelligence; Springer: Berlin, Germany, 2003; Volume 2671, pp. 616-617.

5. Smith, T. Unsupervised neural networks-disruptive technology for seismic interpretation. Oil Gas J. 2010, 108, 42-47. (In English)

6. Rumelhart, D.E.; Hinton, G.E.; Williams, R.J. Learning representations by back-propagating errors. Nature 1986, 323, 533-536. [CrossRef]

7. Hinton, G.E.; Salakhutdinov, R.R. Reducing the Dimensionality of Data with Neural Networks. Science 2006, 313, 504-507. [CrossRef] [PubMed]

8. Nam, S.; Park, H.; Seo, C.; Choi, D. Forged Signature Distinction Using Convolutional Neural Network for Feature Extraction. Appl. Sci. 2018, 8, 153. [CrossRef]

9. Prakash, A.; Moran, N.; Garber, S.; DiLillo, A.; Storer, J. Semantic Perceptual Image Compression using Deep Convolution Networks. In Proceedings of the 2017 Data Compression Conference, Snowbird, UT, USA, 4-7 April 2017; Bilgin, A., Marcellin, M.W., SerraSagrista, J., Storer, J.A., Eds.; IEEE Computer Society: Los Alamitos, CA, USA, 2017.

10. Hu, J.; Xu, X.B.; Pan, X.F.; Liu, L.M. Optimization and Implementation of Image Compression Algorithm Based on Neural Network. In Proceedings of the 2016 6th International Conference on Applied Science, Engineering and Technology, Qingdao, China, 29-30 May 2016; AER-Advances in Engineering Research; Atlantis Press: Paris, France, 2016; Volume 77, pp. 130-136.

11. Jiang, F.; Tao, W.; Liu, S.; Ren, J.; Guo, X.; Zhao, D. An End-to-End Compression Framework Based on Convolutional Neural Networks. IEEE Trans. Circuits Syst. Video Technol. 2017. [CrossRef]

12. Kwon, S.-K.; Jung, H.-S.; Baek, W.-K.; Kim, D. Classification of Forest Vertical Structure in South Korea from Aerial Orthophoto and Lidar Data Using an Artificial Neural Network. Appl. Sci. 2017, 7, 1046. [CrossRef]

13. Gao, F.; Huang, T.; Wang, J.; Sun, J.; Hussain, A.; Yang, E. Dual-Branch Deep Convolution Neural Network for Polarimetric SAR Image Classification. Appl. Sci. 2017, 7, 447. [CrossRef]

14. Nishio, M.; Nagashima, C.; Hirabayashi, S.; Ohnishi, A.; Sasaki, K.; Sagawa, T.; Hamada, M.; Yamashita, T. Convolutional auto-encoder for image denoising of ultra-low-dose CT. Heliyon 2017, 3, e00393. (In English) [CrossRef] [PubMed]

15. Xie, R.; Wen, J.; Quitadamo, A.; Cheng, J.L.; Shi, X.H. A deep auto-encoder model for gene expression prediction. BMC Genom. 2017, 18, 11. [CrossRef] [PubMed]

16. Wang, Y.Q.; Xie, Z.G.; Xu, K.; Dou, Y.; Lei, Y.W. An efficient and effective convolutional auto-encoder extreme learning machine network for 3D feature learning. Neurocomputing 2016, 174, 988-998. (In English) [CrossRef]

17. Vilovic, I. An Experience in Image Compression Using Neural Networks. In Proceedings of the 48th International Symposium ELMAR-2006 Focused on Multimedia Signal Processing and Communications, Zadar, Croatia, 7-10 June 2006; pp. 95-98.

18. Kim, J.; Song, S.; Yu, S.C. Denoising Auto-Encoder Based Image Enhancement For High Resolution Sonar Image. In Proceedings of the 2017 IEEE Underwater Technology (UT), Busan, Korea, 21-24 February 2017; IEEE: New York, NY, USA, 2017. 
19. Qu, J.Y.; Wang, R.B. Collective behavior of large-scale neural networks with GPU acceleration. Cogn. Neurodyn. 2017, 11, 553-563. (In English) [CrossRef] [PubMed]

20. Rizvi, S.T.H.; Cabodi, G.; Francini, G. Optimized Deep Neural Networks for Real-Time Object Classification on Embedded GPUs. Appl. Sci. 2017, 7, 19. [CrossRef]

21. Wang, X.; Zhu, Y.; Huang, L. A comprehensive reconfigurable computing approach to memory wall problem of large graph computation. J. Syst. Archit. 2016, 70, 59-69. [CrossRef]

22. Chen, Y.H.; Krishna, T.; Emer, J.S.; Sze, V. Eyeriss: An Energy-Efficient Reconfigurable Accelerator for Deep Convolutional Neural Networks. IEEE J. Solid State Circuit 2017, 52, 127-138. (In English) [CrossRef]

23. Zhou, Y.M.; Jiang, J.F.; IEEE. An FPGA-based Accelerator Implementation for Deep Convolutional Neural Networks. In Proceedings of the 2015 4th International Conference on Computer Science and Network Technology, Harbin, China, 19-20 December 2015; IEEE: New York, NY, USA, 2015; pp. 829-832.

24. Mansour, W.; Ayoubi, R.; Ziade, H.; Velazco, R.; Falou, W.E. An optimal implementation on FPGA of a hopfield neural network. Adv. Artif. Neural Syst. 2011, 2011. [CrossRef]

25. Liu, Z.Q.; Dou, Y.; Jiang, J.F.; Xu, J.W.; Li, S.J.; Zhou, Y.M.; Xu, Y.N. Throughput-Optimized FPGA Accelerator for Deep Convolutional Neural Networks. ACM Trans. Reconfigurable Technol. Syst. 2017, 10, 23. (In English) [CrossRef]

26. Li, Y.; Ye, X.; Li, Y. Image quality assessment using deep convolutional networks. AIP Adv. 2017, 7, 125324. [CrossRef]

27. Wang, Z.; Simoncelli, E.; Bovik, A.C. Multi-Scale Structural Similarity for Image Quality Assessment. In Proceedings of the Conference Record of the Thirty-Seventh Asilomar Conference on Signals, Systems and Computers, Pacific Grove, CA, USA, 9-12 November 2004.

(C) 2018 by the authors. Licensee MDPI, Basel, Switzerland. This article is an open access article distributed under the terms and conditions of the Creative Commons Attribution (CC BY) license (http:/ / creativecommons.org/licenses/by/4.0/). 